\title{
SINTOMAS DE DEPRESSÃO, RISCO NUTRICIONAL E \\ CAPACIDADE FUNCIONAL EM IDOSOS LONGEVOS
}

Amanda de Jesus dos Santos

Universidade São Judas Tadeu

Daiane Fuga da Silva

Universidade São Judas Tadeu

Ana Lúcia Gatti

Universidade São Judas Tadeu

Adriana Machado Saldiba de Lima

Universidade São Judas Tadeu

Rita de Cássia de Aquino

Universidade São Judas Tadeu

\section{RESUMO}

Objetivou-se avaliar a relação entre sintomas de depressão, risco nutricional e capacidade funcional com características sociais, econômicas, demográficas e de saúde, em idosos longevos. Trata-se de uma pesquisa transversal, realizada em quatro municípios do estado de São Paulo. A relação entre as variáveis foi obtida por meio da técnica de Análise de Correspondência Múltipla. Foram avaliados 103 idosos, a maioria do sexo feminino, com idade entre 80 e 90 anos e com até quatro anos de estudo. Os resultados evidenciam a correspondência entre a presença de sintomas de depressão, risco nutricional, comprometimento funcional, baixa escolaridade e inadequada autoavaliação de saúde. Nota-se, portanto, a necessidade de uma abordagem interdisciplinar, e o desenvolvimento de políticas públicas direcionadas aos muito idosos, oferecendo atenção necessária para a redução do risco de depressão, de desnutrição e na melhoria da funcionalidade dos idosos longevos, que levem a um impacto positivo em sua qualidade de vida.

Palavras-chave: idoso de 80 anos ou mais; depressão; desnutrição. 


\title{
DEPRESSION SYMPTOMS, NUTRITIONAL RISK AND FUNCTIONAL CAPACITY IN LONG- LIVED ELDERLY
}

\begin{abstract}
This is study aimed to evaluate the relationship between depression symptoms, nutritional risk, and functional capacity, with social, economic, demographic and health characteristics in in long-lived elderly. It is a cross-sectional survey carried out in four municipalities in the state of São Paulo. The relationships among the studied variables were investigated through the technique of Multiple Correspondence Analysis. A total of 103 elderly were evaluated, most of whom were female, aged between 80 and 90 years and up to four years of schooling. The results evidence the correspondence between depression symptoms, nutritional risk, functional impairment, low schooling, and inadequate health self-assessment. Therefore, there is need a broad and interdisciplinary approach and the development of public policies directed at the very elderly are necessary, to reduce the risk of depression, malnutrition and improve the functioning of the long-lived elderly, that have a positive impact on their quality of life.
\end{abstract}

Keywords: elderly aged 80 years or more; depression; malnutrition. 


\section{SÍNTOMAS DE DEPRESIÓN, RIESGO NUTRICIONAL Y CAPACIDAD FUNCIONAL EN PERSONAS MAYORES A LARGO PLAZO}

\section{RESUMEN}

Objetivo evaluar la relación entre síntomas de depresión, riesgo nutricional y capacidad funcional, con recursos sociales, económicos, demográficos y de salud, en ancianos de larga vida. Esta es un estudio transversal realizada en cuatro municipios del estado de São Paulo. Las relaciones entre las variables se investigaron utilizando la técnica de Análisis de Correspondencia Múltiple. 103 personas mayores fueron grabadas, la mayoría mujeres, con edades comprendidas entre 80 y 90 años y hasta cuatro años de estudio. Los resultados muestran una correspondencia entre la presencia de síntomas de depresión, riesgo nutricional, deterioro funcional, baja educación y salud inadecuada. Nota la necesidad de un enfoque interdisciplinario y el desarrollo de políticas públicas dirigidas a las personas mayores, ayuda a prestar atención para reducir los riesgos de depresión, desnutrición y mejorar la funcionalidad de las personas mayores de edad, lo que tiene un impacto positivo en su calidad de vida.

Palabras clave: 80 años o más; depresión; desnutrición. 


\section{INTRODUÇÃO}

O aumento de idosos está ocorrendo de forma rápida e progressiva, principalmente nos países em desenvolvimento, como o Brasil, considerado um país em envelhecimento. Observou-se nos últimos anos a diminuição nas taxas de mortalidade, além de aumento na expectativa de vida de 75,3 anos em 2015, para 75,5 anos em 2018. Consequentemente, a população de idosos, definida como indivíduos com 60 anos de idade ou mais, aumentou de 25,4 milhões em 2012 para 30,2 milhões em 2017 (IBGE, 2018).

Os idosos longevos, com 80 anos e mais de vida, podem apresentar características peculiares, tais como maior frequência de doenças crônicas, o que pode contribuir para o aparecimento de dificuldades nas atividades de vida diária, interferindo na independência e autonomia, além de aumentar a tendência ao isolamento, à vulnerabilidade e a fatores de ordem social e psicológica, podendo desencadear sentimentos negativos e culminar em depressão (Lourenço, Lenardt, Kletemberg, Seima, Tallmann, \& Neu, 2012).

Em reconhecimento a sua importância, estudos que avaliam a depressão entre os idosos aumentou globalmente (Nguyen, 2017; Emile, 2017; Pagliai, Vanetti, Caiani, Pasquini, Molino, Cecchi, Sorbi, \& Macchi, 2018). A síndrome depressiva é caracterizada pela presença de humor depressivo ou irritável, e anedonia (diminuição da capacidade de sentir prazer ou alegria), e existe uma sensação subjetiva de fadiga, desinteresse, lentificação, além de pensamentos pessimistas e ideias de ruína. Em geral, esses sintomas são acompanhados de modificações na qualidade do sono, prejuízo cognitivo, alterações comportamentais e sintomas somáticos (Paradela, 2011), comprometendo o apetite e o estado nutricional, podendo acarretar a desnutrição.

A depressão e a desnutrição podem impactar na capacidade funcional do idoso, que condiz sobre a condição que o indivíduo possui de viver de maneira autônoma e de se relacionar em seu meio, sendo uma das formas de avaliar as condições de vida, pois reflete a existência de habilidades físicas e mentais para a manutenção de sua independência (Nogueira, Ribeiro, Rosado, Franceschini, Ribeiro, \& Pereira, 2010; Lassale, Batty, Baghdadii, Jacka, Sánchez-Villegas, Kivimäki, \& Akbaraly, 2018). Para a saúde do idoso longevo, a capacidade funcional representa manter sua autonomia em viver sozinho e desenvolver atividades que the proporcione prazer. Pode ser entendida como a capacidade de qualquer indivíduo se adaptar aos problemas cotidianos, apesar de possuir limitações física, mental ou social, e a possibilidade de cuidar de si mesmo, de determinar e executar as atividades de vida diária (Sobral, Araújo, \& Sobral, 2018).

A tríade depressão-desnutrição-perda de funcionalidade pode ser observada em idosos sem ser possível identificar a causa e o efeito. A identificação de sintomas de depressão e risco nutricional é relevante à medida que implica em diversas 
consequências que afetam a vida do idoso, podendo afetar sua capacidade funcional, tornando-o fragilizado (Fhon, 2018).

Assim, o objetivo deste trabalho foi avaliar a relação entre sintomas de depressão, risco nutricional e capacidade funcional, com características sociais, econômicas, demográficas e de saúde, em idosos longevos.

\section{MÉTODO}

Este estudo é parte do projeto intitulado "Padrões de envelhecimento físico, cognitivo e psicossocial em idosos longevos que vivem em diferentes contextos", proposto pela Universidade Estadual de Campinas (UNICAMP) e realizado em parceria com a Universidade São Judas Tadeu, aprovado pelo CEP desta instituição, com o número do CAAE: 56493316.0.0000.0089 do parecer $n^{\circ} 1.693 .318$.

\section{TIPO DE ESTUDO}

Estudo do tipo transversal descritivo, realizado entre outubro de 2016 e outubro de 2017.

\section{LOCAL DE ESTUDO}

A coleta de dados ocorreu em quatro instituições localizadas no estado de São Paulo: Casa do Idoso Norte (São José dos Campos), CISE (Centro Integrado de Saúde e Educação da Terceira Idade) Moacyr Rodrigues (São Caetano do Sul), Escola Superior de Educação Física (Jundiaí) e na Universidade São Judas. As instituições localizadas em São José dos Campos, São Caetano do Sul e Jundiaí são consideradas centros de referência do idoso por apresentarem espaços de uso exclusivos para maiores de 60 anos, onde é oferecido atendimento médico ambulatorial, aliados à espaços de convivência.

\section{AMOSTRA E CASUÍstica}

Trata-se de amostra não probabilística por conveniência de 103 idosos longevos, residentes no estado de São Paulo. Foram incluídos todos os idosos com idade maior de 80 anos frequentadores das instituições descritas, que atenderam aos critérios de inclusão e concordaram em participar.

\section{CRITÉRIOS DE INCLUSÃO E EXCLUSÃO}

Foram incluídos no estudo idosos de ambos os sexos, com idade maior de 80 anos e capazes de se comunicarem, e excluídos os idosos que apresentarem dificuldade de compreensão para o fornecimento das informações necessárias para o estudo.

\section{PROCEDIMENTOS}


Os dados foram levantados por uma equipe multiprofissional composta por psicólogos, nutricionistas e fisioterapeutas, devidamente treinados e padronizados para as técnicas de coleta e avaliação.

Os participantes selecionados assinaram um Termo de Consentimento Livre e Esclarecido - TCLE desenvolvido a partir da Resolução n466/2012. Cabe ressaltar que os dados foram obtidos em local apropriado, com duração aproximada de duas horas de coleta. O caderno de coleta foi composto por vinte e dois blocos. O primeiro bloco incluía dados pessoais, sociodemográficos (sexo, idade, escolaridade, cor, estado civil, renda, condições de saúde, moradia e suporte social), dados de saúde e psicossociais, além de instrumentos de avaliação de qualidade de vida, sintomas de depressão, risco nutricional e capacidade funcional.

Os instrumentos do caderno utilizados no presente estudo foram: Escala de Depressão Geriátrica (GDS-15), Mini Avaliação Nutricional (MAN), conjunto de questões relativas às atividades de vida diária (AVD), atividades avançadas de vida diária (AAVD), atividades instrumentais de vida diária (AIVD), atividades básicas de vida diária (ABVD).

A Escala de Depressão Geriátrica (GDS-15) é um instrumento de rastreio reconhecido como recurso rápido, simples e útil para identificação de sintomas depressivos ou de vulnerabilidade à depressão em idosos. A versão abreviada é composta por 15 questões e foi elaborada por Sheikh e Ysavage (1986) (Paradela, Lourenço, \& Veras, 2005). A escala possui itens com respostas dicotômicas, onde é assinalada a presença ou a ausência (sim/não) de sintomas referentes a mudança de humor e a sentimentos específicos como desamparo, inutilidade, desinteresse, aborrecimento e felicidade. A obtenção de 0 a 5 pontos indica a ausência de depressão, de 6 a 10 pontos indica depressão leve e de 11 a 15 pontos indica depressão grave. Suas vantagens incluem a rápida e fácil aplicação, além de não necessitar de um profissional da área da saúde mental para sua aplicação (Batistoni, Neri, \& Cupertino, 2007).

A Mini Avaliação Nutricional (MAN) é uma ferramenta que foi desenvolvida em uma parceria entre o Hospital Universitário de Toulouse, França, a Universidade do Novo México (USA) e a Nestlé Research Center (Suiça). O objetivo da ferramenta é rastrear o risco de desenvolver a desnutrição ou detectá-la em estágio inicial, e inclui aspectos físicos e mentais, que podem afetar a ingestão alimentar dos idosos, e um questionário dietético. Consiste em um questionário dividido em duas partes, sendo a primeira denominada Triagem Nutricional, e a segunda denominada Avaliação Global. A Triagem compreende as seguintes questões: 1) alteração da ingestão alimentar, 2) perda de peso nos últimos meses, 3) avaliação de mobilidade, 4) presença de estresse psicológico ou doença aguda no último trimestre, 5) problemas neuropsicológicos e 6) Índice de Massa Corporal (IMC). Para cada item é atribuído um escore, e se a pontuação final for igual ou inferior a 11 pontos significa que há a possibilidade de desnutrição. A avaliação global, ou segunda parte da avaliação, aborda questões relacionadas ao modo de vida, 
presença de lesões de pele, uso de medicações, inquérito dietético (número de refeições, ingestão de alimentos e líquidos, capacidade autônoma de alimentação), autoavaliação da saúde e estado nutricional, e dados antropométricos (perímetro braquial e da panturrilha). Ao final, o somatório dos pontos indica se o idoso se apresenta sem risco nutricional (escore maior que 23,5 pontos), em risco nutricional (escore de 17 a 23,5 pontos) ou desnutrido (escore menor que 17 pontos) (Rubenstein, Harker, Salva, Guigoz, \& Vellas, 2001).

As Atividades Básicas de Vida Diária (ABVD) foram avaliadas a partir da escala de Katz, desenvolvida em 1963 por Sidney Katz, a qual possui seis itens que medem o desempenho do indivíduo nas atividades de autocuidado, os quais obedecem a uma hierarquia de complexidade da seguinte forma: alimentação, controle esfincteriano, transferência (mobilidade), autonomia para ir ao banheiro, capacidade para se vestir e tomar banho. As Atividades Instrumentais de Vida Diária (AIVD) foram identificadas por meio da escala desenvolvida por Lawton e Brody, em 1969. As AIVD comportam tarefas adaptativas e necessárias para vida independente na comunidade: preparo de refeições, realização de compras, utilização de transporte, tarefas domésticas, uso do telefone, manejo do dinheiro e utilização de medicamentos. Foram considerados independentes os idosos que não necessitavam de assistência para nenhuma ABVD e AIVD, dependentes parciais aqueles que necessitavam de assistência em, pelo menos, uma ABVD e AIVD, e dependentes totais os que necessitavam de assistência em todas as ABVD e AIVD (Alves, Leite, \& Machado, 2008; Muniz, Aguiar, Brito, Freitas, Moreira, \& Araújo 2016).

As Atividades Avançadas de Vida Diária (AAVD) foram mensuradas a partir de escala desenvolvida por Reuben et al. (1990) e essas atividades dizem respeito a independência na vida prática, no trabalho, no lazer e em outros papeis sociais comuns. Foram considerados como mais ativos aqueles que realizavam quatro ou mais atividades e, como menos ativos, os idosos que relataram realizar três ou menos atividades (Oliveira, Silva, Lopes, Cachioni, Falcão, Batistoni, Neri, \& Yassuda, 2015).

\section{ANÁLISE DOS DADOS}

Buscou-se explorar relações conjuntas entre as categorias das variáveis investigadas por meio da Análise de Correspondência Múltipla (ACM). É uma técnica multivariada de interdependência, de caráter exploratório, apropriada para situações nas quais se deseja analisar dados categóricos, com visualização de associações entre linhas e colunas de uma matriz. Ao realizar o cruzamento entre linhas e colunas, obtém-se um perfil definido do conjunto de dados, tornando-se possível representar graficamente as relações entre categorias de variáveis (Silva, Souza, Silva, Petroski, \& Fonseca, 2015).

Na ACM não há definido um procedimento de seleção das variáveis para compor a análise multivariada. A seleção é realizada com base na subjetividade do pesquisador e no conhecimento teórico acerca do assunto analisado. Nesse 
método as categorias das variáveis estudadas são representadas visualmente pelos mapas perceptuais das dimensões, e sua correspondência é avaliada de acordo com a proximidade entre as categorias. Quanto mais próximas duas categorias no gráfico, mais frequente é a sua ocorrência conjunta.

O primeiro passo da análise é a determinação do número de dimensões. No presente estudo optou-se por duas dimensões pelo princípio da parcimônia, pois explicavam a maior parte da variância. As medidas de discriminação informam sobre as variáveis que mais contribuem para definição de cada uma das dimensões, sendo estas as mais relevantes em termos de interpretação gráfica. Esta medida determina em qual dimensão a variável se localiza, e os valores devem ser próximos ou superiores ao valor de inércia da dimensão observado. Após determinada em qual dimensão a variável se encontra, a ACM permite a obtenção das coordenadas dos centroides (CC) de cada variável. Esses valores indicam a localização gráfica das categorias e são acompanhas por sinais positivos e negativos, que permitem identificar a posição em relação às dimensões, e categorias que se associam possuem o mesmo sinal, e as que se opõem possuem sinais contrários.

O método não permite estabelecer a significância estatística das associações, nem avaliar o efeito independente de cada característica. Porém, combina as vantagens de métodos não lineares e multidimensionais, e os dados são processados e analisados estatisticamente (Silva et al., 2015). A análise foi realizada pelo programa Statistical Package for the Social Sciences (SPSS), versão 20.0.

Uma vez que a seleção é realizada com base na subjetividade do pesquisador e no conhecimento teórico acerca do assunto analisado, foram selecionadas as seguintes variáveis para análise: GDS (15), MAN, ABVD, AIVD, AAVD, autoavaliação da saúde, morar sozinho, escolaridade, disponibilidade de cuidador (se necessário) e dinheiro suficiente para cuidados básicos.

\section{RESULTADOS}

No presente estudo foram avaliados 103 idosos, com idade média de 82 anos ( \pm 2,96 anos), sendo a maioria do sexo feminino $(68,9 \%), 52,4 \%$ dos idosos relataram baixa escolaridade (até quatro anos de estudo) e 58,3\% viúvos.

Com relação aos sintomas de depressão, risco nutricional e avaliação de funcionalidade, observou-se $26,2 \%$ idosos com sintomas de depressão leve, $28,2 \%$ em risco nutricional e, segundo as atividades de vida diária, 85,4\% eram mais ativos (AAVD), 49,5\% dependentes parciais (AIVD) e 66,0\% independentes (ABVD) (Tabela 1).

\section{Tabela 1}

Frequência observada nos instrumentos MAN-Completa, GDS-15, AAVD, AIVD e ABVD (n=103). São Paulo, 2017. 


\begin{tabular}{|c|c|c|}
\hline Instrumentos & $\mathbf{n}$ & $\%$ \\
\hline \multicolumn{3}{|c|}{ GDS-15 (Sintomas de depressão) } \\
\hline Depressão grave & 1 & 1,0 \\
\hline Depressão leve & 27 & 26,2 \\
\hline Sem depressão & 73 & 70,9 \\
\hline NR & 2 & 1,9 \\
\hline \multicolumn{3}{|c|}{ MAN-Completa (Risco Nutricional) } \\
\hline Desnutrição & 0 & 0,0 \\
\hline Em risco nutricional & 29 & 28,2 \\
\hline Sem risco nutricional & 73 & 70,9 \\
\hline NR & 1 & 1,0 \\
\hline \multicolumn{3}{|l|}{ AAVD } \\
\hline Mais ativo & 88 & 85,4 \\
\hline Menos ativo & 13 & 12,4 \\
\hline NR & 2 & 1,9 \\
\hline \multicolumn{3}{|l|}{ AIVD } \\
\hline Independente & 51 & 49,5 \\
\hline Dependente parcial & 51 & 49,5 \\
\hline Dependente total & 0 & 0,0 \\
\hline NR & 1 & 1,0 \\
\hline \multicolumn{3}{|l|}{$A B V D$} \\
\hline Independente & 68 & 66,0 \\
\hline Dependente parcial & 34 & 33,0 \\
\hline Dependente total & 0 & 0,0 \\
\hline NR & 1 & 1,0 \\
\hline Total & 103 & 100 \\
\hline
\end{tabular}

Nota. NR: Não Respondeu; MAN-Completa: Mini Avaliação Nutricional-Completa; GDS-15: Escala de Depressão Geriátrica; AAVD: Atividades Avançadas de Vida Diária; AIVD: Atividades Instrumentais de Vida Diária; ABVD: Atividades Básicas de Vida Diária.

Com relação às outras variáveis inseridas na Análise de Correspondência Múltipla (ACM) observou-se que $35,9 \%$ dos idosos avaliaram sua saúde como ruim ou regular, 34,0\% moravam sozinhos, 2,9\% não teriam cuidadores ou familiares disponíveis para cuidado, caso necessitassem, e 20,4\% relataram não possuir dinheiro suficiente para cobrir as suas necessidades básicas (Tabela 2). 
Tabela 2

Frequência observada das variáveis inseridas na Análise de Correspondência Múltipla (ACM) dos idosos longevos ( $n=103)$. São Paulo, 2017.

\begin{tabular}{|c|c|c|}
\hline Variáveis & $\mathbf{n}$ & $\%$ \\
\hline \multicolumn{3}{|c|}{ Autoavaliação da saúde } \\
\hline Ruim & 2 & 1,9 \\
\hline Regular & 35 & 34,0 \\
\hline Boa & 49 & 47,6 \\
\hline Muito boa & 16 & 15,5 \\
\hline NR & 1 & 1,0 \\
\hline \multicolumn{3}{|l|}{ Mora sozinho } \\
\hline Sim & 35 & 34,0 \\
\hline Não & 68 & 66,0 \\
\hline \multicolumn{3}{|c|}{ Cuidador, se necessário } \\
\hline Sim & 82 & 79,6 \\
\hline Não & 3 & 2,9 \\
\hline NR & 18 & 17,5 \\
\hline \multicolumn{3}{|c|}{$\begin{array}{l}\text { Dinheiro suficiente para cobrir necessidades } \\
\text { básicas }\end{array}$} \\
\hline Sim & 80 & 77,7 \\
\hline Não & 21 & 20,4 \\
\hline NR & 2 & 1,9 \\
\hline \multicolumn{3}{|c|}{ Escolaridade (anos de estudo) } \\
\hline Nenhum & 11 & 10,7 \\
\hline 1 a 4 anos & 54 & 52,4 \\
\hline 5 a 11 anos & 27 & 26,2 \\
\hline Mais de 11 anos & 11 & 10,7 \\
\hline Total & 103 & 100 \\
\hline
\end{tabular}

Nota: NR: Não respondeu; *Está relacionado em ter alguém para ajudar a realizar as atividades de vida diária (ABVD, AIVD e AAVD), caso venha a necessitar.

Determinou-se, pelo princípio da parcimônia, o número de duas dimensões, observadas a partir da horizontalização do decréscimo dos autovalores, constatando-se que valores maiores que 1,5 explicam $23,9 \%(0,239)$ e $19,2 \%(0,192)$ das variâncias, respectivamente.

Para a construção do modelo, empregaram-se medidas de discriminação das variáveis. A seleção das variáveis para o modelo foi definida por meio das medidas 
de discriminação, isto é, aquelas próximas ou superiores aos valores de inércia da dimensão $1(0,239)$ e da inércia da dimensão $2(0,192)$. A quantificação das categorias das variáveis nas dimensões do modelo ocorreu por meio de observação dos valores de suas coordenadas de centroides (CC), que permitem a construção da figura por dimensão pelas cargas positivas e negativas de cada categoria (Tabela 3).

Há uma distribuição semelhante na contribuição de cada variável na composição da primeira e da segunda dimensão. Na primeira dimensão as categorias constatadas foram: presença de sintomas de depressão, atividades avançadas de vida diária inativo, atividades instrumentais de vida diária dependente (AIVD) autoavaliação da saúde regular (AAS) e baixa escolaridade (Bescol), e na segunda dimensão as categorias constatadas foram risco nutricional presente (MAN) e a variável não morar sozinho (MS) (Tabela 3).

Tabela 3

Medidas de discriminação das variáveis e coordenadas dos centroides nas dimensões (1 e 2), segundo as categorias do modelo final, observadas entre idosos longevos $(n=103)$. São Paulo, 2017.

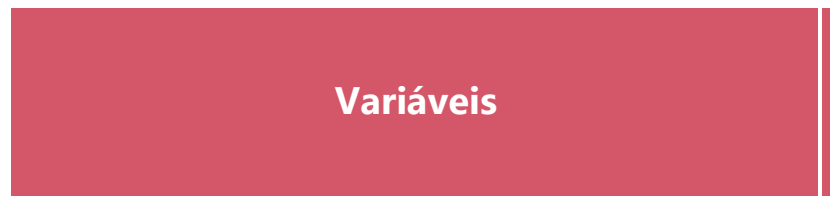

\section{Medida de Discriminação (MD)} Dimensões
Escala de Depressão Geriátrica ${ }^{a}$

Com sintomas de depressão

Sem sintomas depressão

Mini Avaliação Nutricional ${ }^{a}$

Com risco nutricional

Sem risco nutricional

Atividades Avançadas de Vida Diária

Mais ativo

Inativo

Atividades Instrumentais de Vida Diária

Independente

Dependente

\section{$0,343 \quad 0,672$}

$0,135 \quad 0,259$

$0,410 \quad 0,171$

1,015

0,663

$-0,41$

0,258

$0,119 \quad 0,040$

- $\quad 0,075$

0,129

$0,913 \quad 0,525$

$0,270 \quad 0,020$

0,525

Coordenadas

(CC) 


\begin{tabular}{|c|c|c|c|c|}
\hline Autoavaliação da saúde ${ }^{a}$ & 0,601 & 0,486 & & \\
\hline Ruim & & & 2,648 & 0,087 \\
\hline Regular & & & 0,644 & 0,620 \\
\hline Boa & & & - & - \\
\hline Boa & & & 0,069 & 0,718 \\
\hline Muito boa & & & $-1,44$ & 0,842 \\
\hline Baixa Escolaridade & 0,412 & 0,059 & & \\
\hline Sim & & & 0,494 & - \\
\hline $\sin$ & & & - & 0,195 \\
\hline Não & & & 0,836 & 0,307 \\
\hline Dinheiro suficiente ${ }^{a}$ & 0,048 & 0,360 & & \\
\hline Sim & & & 0,080 & - \\
\hline ำก & & & - & 0,294 \\
\hline NaO & & & 0,461 & 1,199 \\
\hline Morar sozinhoa & 0,009 & 0,228 & & \\
\hline Sim & & & 0,136 & - \\
\hline Não & & & - & 0,676 \\
\hline & & & 0,065 & 0,333 \\
\hline
\end{tabular}

Nota: a Variáveis (e, por conseguinte, categorias) cujas medidas de discriminação foram próximas ou superiores aos valores de inércia da dimensão 1 e 2 (0,239 e 0,192). 
As relações entre categorias de variáveis e a formação de conglomerados foram avaliadas analisando-se proximidade (Figura 1) e a formação de um Mapa Perceptual, que caracteriza a Análise de Correspondência Múltipla. As variáveis que se aproximaram propiciaram a descrição de um perfil de idosos com presença de sintomas de depressão (GDSs), presença de risco nutricional (MANs), atividades avançadas de vida diária inativo (AAVDi), atividades instrumentais de vida diária dependente (AIVDd), autoavaliação da saúde regular (AASre) e baixa escolaridade (Bescols).

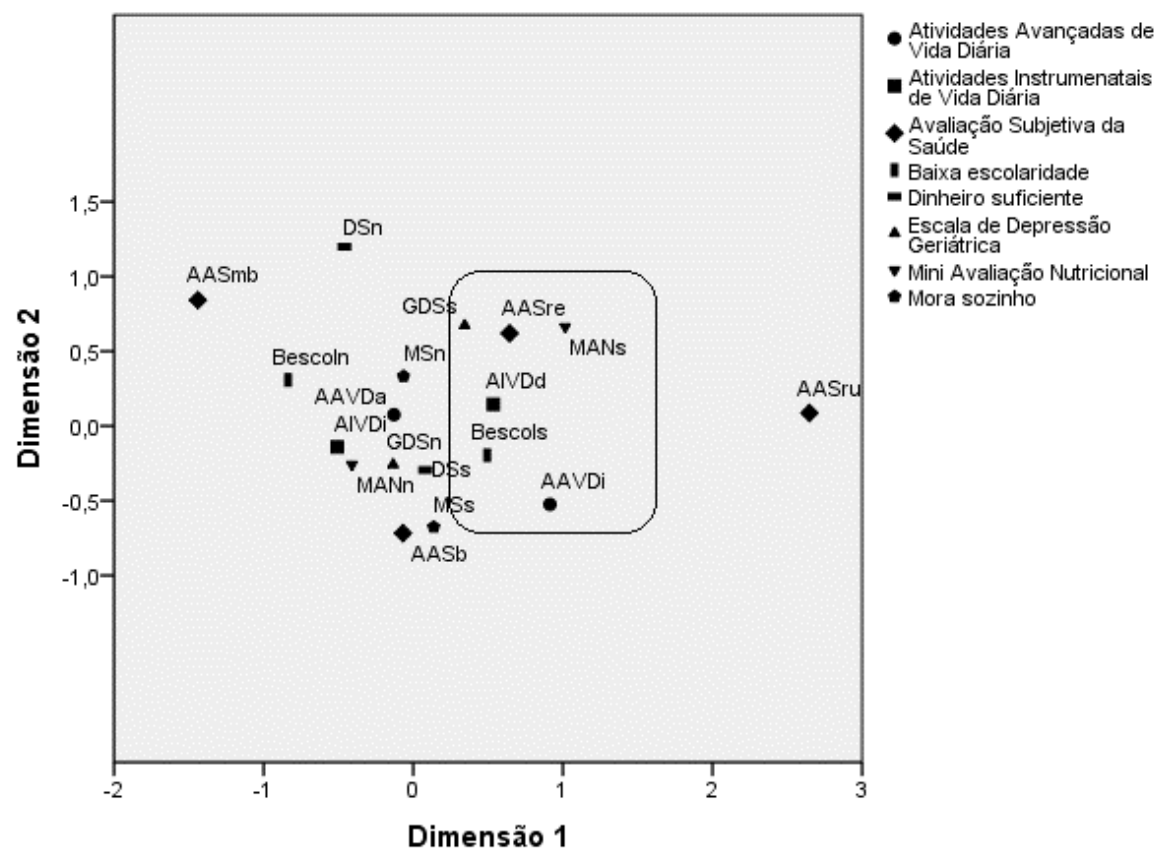

Figura 1. Mapa perceptual entre as categorias das variáveis e formação de conglomerados.

Nota: Atividades Avançadas de Vida Diária: AAVDa (ativo) e AAVDi (inativo); Atividades Instrumentais de Vida Diária: AIVDi (independente) e AIVDd (dependente); Auto Avaliação da Saúde: AASmb (muito boa), AASb (boa), AASre (regular) e AASru (ruim); Baixa escolaridade: Bescols (sim) e Bescoln (não); Dinheiro suficiente: DSs (sim) e DSn (não); Sintomas de Depressão Geriátrica: GDSs (GDS-sim) e GDSn (GDS-não); Mini Avaliação Nutricional: MANs (MAN-sim/com risco) e MANn (MAN- não/sem risco) Mora sozinho: MSs (sim) e MSn (não).

\section{DISCUSSÃO}

No presente estudo observou-se que cerca de $30 \%$ dos idosos apresentavam sintomas de depressão e risco nutricional, e constatou-se a correspondência com comprometimentos de funcionalidade (atividades avançadas de vida diária inativo e atividades instrumentais de vida diária dependente), além de baixa escolaridade e autoavaliação regular da saúde. Os sintomas de depressão e o risco nutricional se apresentaram relacionados em estudos internacionais. No trabalho de 
Bartoszek, Domżał-Drzewicka, Kachaniuk, Kocka e Muzyczka (2015) observou-se associação entre a presença de sintomas de depressão grave e risco nutricional entre os que relataram não possuir dinheiro suficiente para todas as suas necessidades. Dent, Wright, Hoogendijk e Hubbard (2018) observaram que idosos desnutridos eram mais propensos a sintomas de depressão, além de serem frágeis e dependentes para realizar as atividades instrumentais de vida diária. No estudo italiano MUGELLO, realizado com idosos nonagenários, foi observada associação entre sintomas de depressão, baixo peso e baixa escolaridade (Pagliai et al., 2018).

A identificação de sintomas de depressão frequentemente está relacionada ao estado nutricional, uma vez que interfere no centro de controle neural, que é responsável pela fome, ansiedade e compulsões alimentares, e pode ser apontada como uma das causas de ganho ou perda de peso (Nóbrega, Leal, Marques, \& Vieira, 2015).

Keshavarzi, Ahmadi e Lankarani (2014) constataram que os idosos desnutridos ou em risco nutricional exibiram pontuações superiores de GDS, além de apresentaram menor nível de escolaridade. A baixa escolaridade foi prevalente em mais da metade do presente estudo, provavelmente devido às dificuldades de acesso ao ambiente escolar no passado, associadas às atividades agrícolas, operárias e de dona de casa, fato convergente com a informação levantada pelo censo de 2014 quanto à baixa escolaridade dos idosos responsáveis pelos domićlios no Brasil (64,8\%) (IBGE, 2014). Analfabetismo e baixa escolaridade representam uma realidade em países em desenvolvimento, pois os idosos viveram sua infância e adolescência em uma época em que o ensino não era prioritário, podendo acarretar a velhice maior dependência física e econômica (Pilger, Menon \& Mathias, 2013; Rocha, Oliveira, \& Gardenghi, 2017).

Em estudos nacionais houve similaridade entre os resultados encontrados, pois mostraram que a baixa escolaridade, quando associado às condições econômicas precárias e à dificuldade de acesso a rede de suporte, pode trazer em idosos dificuldades no autocuidado (Borges, Santos, Kummer, Fior, Molin, \& Wibelinger, 2014; Güths, Jacob, Santos, Arossi, \& Béria, 2017). Oliveira, Bezerra, Silva, Alves, Moreira, \& Caldas (2012) e Magalhães, Carvalho, Carvalho, Alencar, Moreira, \& Parente (2016) afirmam que a escolaridade mais elevada é um fator protetor para o idoso, possivelmente pelo melhor conhecimento, mais acesso a medidas preventivas e melhor situação financeira. Estudo realizado por Cohen, Paskulin e Prieb (2015) observou que $36,5 \%$ dos idosos atendidos em serviço de emergência apresentavam sintomas depressivos, e estes foram associados a ser viúvo, sendo que a frequência de sintomas de depressão foi maior entre idosos com menor escolaridade e do sexo feminino, que não utilizavam serviços de saúde.

O sintoma de depressão associou-se à incapacidade funcional para realização das atividades de vida diária. Vários estudos confirmam este achado, como o de Tavares et al. (2016), que observaram que a incapacidade funcional para realização das atividades instrumentais de vida diária (AIVD) foi associada a sintomas de 
depressão, idade acima de 80 anos, baixa renda, baixa escolaridade e presença de cinco ou mais doenças. No trabalho de Silva, Barbosa, Pinho, Figueiredo, Amaral, Cunha, Alves e Barbosa (2018) os idosos com idade acima de 80 anos e que viviam sem companheiro, tiveram o triplo de chance de apresentar níveis mais elevados de dependência funcional, hipotetizando-se que o idoso, ao perder a sua funcionalidade, estará mais propício ao isolamento social, o que poderá impactar em suas relações pessoais e acarretar sintomas depressivos. No estudo italiano MUGELLO foi observada associação entre sintomas de depressão, viuvez e incapacidade funcional para realização de atividades básicas e instrumentais (Pagliai et al., 2018).

No presente estudo houve associação entre ser menos ativo na realização das atividades avançadas de vida diária (AAVD) e a presença de sintomas de depressão. O estudo FIBRA, realizado no distrito de Ermelino Matarazzo (SP), observou que idosos que eram menos ativos apresentaram pior desempenho cognitivo, bem como maiores pontuações no GDS, indicativo de sintomas depressivos (Oliveira et al., 2015). No estudo de Aguiar, Marques, Silva, Costa, Ramos e Leal (2014) os idosos independentes nas atividades instrumentais de vida diária (AIVD) não apresentavam sintomas sugestivos de depressão.

A autoavaliação regular da saúde relacionou-se com o GDS. Aguiar et al. (2014) observaram associação entre autoavaliação da saúde e sintomas de depressão, de tal forma que, ao declarar uma boa saúde, tem-se um percentual muito baixo de idosos com GDS maior que 5, mas ao declarar sua saúde ruim, o percentual de idosos aumentou de 2,0\% para 23,6\%. No estudo de Borges et al. (2014) a autoavaliação da saúde atual foi considerada regular por $34,7 \%$ da população idosa estudada, dados que corroboram os achados do presente estudo (34,0\%). A autoavaliação da saúde é um indicador relevante do seu bem-estar, útil para avaliar o atendimento a necessidades globais do idoso, qualidade de vida e para predizer sobrevida.

No presente estudo optou-se por não estudar separadamente as variáveis sexo masculino e feminino e avaliar os sintomas de depressão, risco nutricional e capacidade funcional no grupo de idosos longevos independentemente do gênero. No entanto, Payahoo, Khaje-Bishak, Pourghassem, Kabir-Alavi e Asgharijafarabadi (2013) demonstraram correlação positiva entre os instrumentos MAN e GDS em ambos os sexos, mas participantes do sexo feminino apresentavam cinco vezes mais sintomas de depressão do que os idosos do sexo masculino. Santos, Ikegami, Almeida, Paiva e Silveira (2016) observaram que mulheres apresentaram maior nível de dependência nas atividades básicas de vida diária (ABVD). Em trabalhos de prevalência de incapacidade funcional, as taxas são maiores em mulheres, mas ocorre devido sua maior longevidade em comparação aos homens, uma vez que, com o avanço da idade, existe maior probabilidade de dependência nas atividades de vida diária (Pereira, Bastos, Del Duca, \& Bós, 2012) 
É importante destacar as limitações do presente estudo, como a possibilidade de desejabilidade social, que é uma das formas de enviesamento mais comuns em contexto de avaliação psicológica. Ao estudar depressão e autoavaliação da saúde, o idoso pode dar respostas não verídicas aos itens das escalas, no sentido de apresentarem uma imagem positiva de si próprio. Outra limitação está relacionada a amostra que incluiu apenas idosos que vivem em comunidade e se deslocam para atividades consideradas avançadas para a vida diária, e não foram incluídos idosos longevos que vivem isolados em domicílios ou instituições de longa permanência. Além disso, a amostra de conveniência e a coleta transversal limitam a interpretação dos dados.

No entanto, o estudo incluiu uma amostra importante de longevos, foi desenvolvido sob o aspecto interdisciplinar, e permitiu correlacionar os fatores com uma técnica estatística (Análise de Correspondência Múltipla) que combina as vantagens de métodos não lineares e multidimensionais.

\section{CONSIDERAÇÕES FINAIS}

Os principais achados do presente estudo foram a correspondência entre a presença de sintomas depressão, risco nutricional, comprometimento funcional, baixa escolaridade e inadequada autoavaliação de saúde. Nota-se, portanto, a necessidade de desenvolvimento de uma abordagem ampla e interdisciplinar, e o desenvolvimento de políticas públicas direcionadas aos muito idosos, oferecendo atenção necessária para a redução do risco de depressão, de desnutrição e na melhoria da funcionalidade dos idosos longevos, que levem a um impacto positivo em sua qualidade de vida. Sugere-se que estudos futuros possam implementar programas interventivos com o intuito de colaborar com desenvolvimento de ações direcionadas aos longevos, oferecendo acompanhamento interdisciplinar e atenção necessária que impacte em sua qualidade de vida, especificamente, trabalhando na redução do risco de depressão, desnutrição e na melhoria da funcionalidade.

\section{REFERÊNCIAS}

Aguiar, A. M. A., Marques, A. P. O., Silva, E. C., Costa, T. R., Ramos, R. S. Pe. S., \& Leal, M. C. C. (2014) Prevalência e determinantes de sintomatologia depressiva em idosos assistidos em serviço ambulatorial. Revista Brasileira de Geriatria e Gerontologia, 17(4), 853-866. https://dx.doi.org/10.1590/1809-9823.2014.13198

Alves, L. C., Leite, I. C., \& Machado, C. J. (2008). Conceituando e mensurando a incapacidade funcional da população idosa: uma revisão de literatura. Ciência \& Saúde Coletiva, 13(4), 1199-1207. https://dx.doi.org/10.1590/S1413-81232008000400016

Bartoszek, A., Domżał-Drzewicka, R., Kachaniuk, H., Kocka, K., \& Muzyczka, K. (2015). The state of nutrition and the self-assessment of symptoms of depression in the group of seniors living in the countryside of Lublin province - preliminary report. Przeglad gastroenterologiczny, 10(4), 208-214. Recuperado de https://www.ncbi.nlm.nih.gov/pubmed/26759627

Batistoni, S. S. T., Neri, A. L., \& Cupertino, A. P. F. B. (2007). Validade da escala de depressão do Center for Epidemiological Studies entre idosos brasileiros. Revista de Saúde Pública, 41(4), 598-605. https://dx.doi.org/10.1590/S0034-89102007000400014

Borges, A. M., Santos, G., Kummer, J. A., Fior, L., Molin, V. D., \& Wibelinger, L. M. (2014). Autopercepção de saúde em idosos residentes em um município do interior do Rio Grande do Sul. Revista 
Brasileira de Geriatria e Gerontologia, 17(1), 79-86. https://dx.doi.org/10.1590/S1809$\underline{98232014000100009}$

Cohen, R., Paskulin, L. M. G., \& Prieb, R. G. G. (2015). Prevalência de sintomas depressivos entre idosos em um serviço de emergência. Revista Brasileira de Geriatria e Gerontologia, 18(2), 307-317. https://dx.doi.org/10.1590/1809-9823.2015.14052

Dent, E., Wright, O., Hoogendijk, E.O., \& Hubbard, R.E. (2018). Nutritional screening and dietitian consultation rates in a geriatric evaluation and management unit. Nutrition \& Dietetics, 75(1), 11-16. http://dx.doi.org/10.1111/1747-0080.12391.

Emile, P-M., Priscila R. F. C., Valterlinda, A. O. Q., Marcos, P-S., \& Mônica, L. P. S. (2017): Overweight and Obesity Associated with Higher Depression Prevalence in Adults: A Systematic Review and Meta-Analysis. Journal of the American College of Nutrition, 36(3), 223-233. http://dx.doi.org/10.1080/07315724.2016.1261053

Fhon, J. R. S., Rodrigues, R. A. P., Santos, J. L. F., Diniz, M. A., Santos, E. B., Almeida, V. C., \& Giacomini, S. B. L. (2018). Factors associated with frailty in older adults: a longitudinal study. Revista de Saúde Pública, 52(74). https://dx.doi.org/10.11606/s1518-8787.2018052000497

Furihata, R., Konno, C., Suzuki, M., Takahashi, S., Kaneita, Y., Ohida, T., \& Uchiyama, M. (2018) Unhealthy lifestyle factors and depressive symptoms: A Japanese general adult population

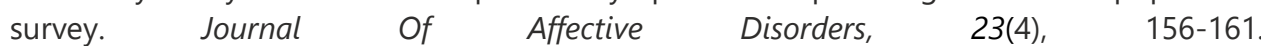
http://dx.doi.org/10.1016/j.jad.2018.02.093.

Güths, J. F.S., Jacob, M. H. V. M., Santos, A. M. P. V., Arossi, G. A., \& Béria, J. U. (2017). Sociodemographic profile, family aspects, perception of health, functional capacity and depression in institutionalized elderly persons from the north coastal region of Rio Grande do Sul, Brazil. Revista Brasileira de Geriatria e Gerontologia, 20(2), 175-185. https://dx.doi.org/10.1590/1981-22562017020.160058

Instituto Brasileiro de Geografia e Estatística. (2014). Perfil dos estados e municípios brasileiros. Recuperado de https://ww2.ibge.gov.br/home/estatistica/economia/perfilmunic/2014/

Instituto Brasileiro de Geografia e Estatística. (2018). Número de idosos cresce 18\% em 5 anos e ultrapassa 30 milhões 2017.2 Recuperado de https://agenciadenoticias.ibge.gov.br/agencia-noticias/2012-agencia-de

noticias/noticias/20980-numero-de-idosos-cresce-18-em-5-anos-e-ultrapassa-30-milhoes em-2017

Keshavarzi, S., Ahmadi, S. M., \& Lankarani, K. (2014). The Impact of Depression and Malnutrition on Health-Related Quality of Life Among the Elderly Iranians. Global Journal of Health Science, 7(3), 161-170. Recuperado de https://www.ncbi.nlm.nih.gov/pmc/articles/PMC4802080/\#

Lawton, M. P., Brody, E. M. (1969). Assessment of Older People: Self-Maintaining and Instrumental Activities of Daily Living. The Gerontologist, 9(3), 179186. https://dx.doi.org/10.1093/geront/9.3 Part 1.179

Lourenço, T. M., Lenardt, M. H., Kletemberg, D. F., Seima, M. D., Tallmann, A. E. C., \& Neu, D. K. M. (2012). Capacidade funcional no idoso longevo: uma revisão integrativa. Revista Gaúcha de Enfermagem, 33(2), 176-185. https://dx.doi.org/10.1590/S1983-14472012000200025

Magalhães, J. M., Carvalho, A. M. B., Carvalho, S. M.; Alencar, D. C., Moreira, W. C., \& Parente, A. C. M. (2016). Depressão em idosos na estratégia saúde da família: uma contribuição para a atenção primária. Revista Mineira de Enfermagem, 20. Recuperado de http://www.reme.org.br/artigo/detalhes/1080

Muniz, E., Aguiar, M., Brito, M., Freitas, C., Moreira, A., \& Araújo, C. (2016). Desempenho nas atividades básicas da vida diária de idosos em Atenção Domiciliar na Estratégia Saúde da Família. Revista Kairós: Gerontologia, 19(2), 133-146. https://doi.org/10.23925/2176901X.2016v19i2p133-146

Nóbrega, I. R. A. P., Leal, M. C. C., Marques, A. P. O., \& Vieira, J. C. M. (2015). Fatores associados à depressão em idosos institucionalizados: revisão integrativa. Saúde Debate, 39(105), 536550. https://doi.org/10.1590/0103-110420151050002020

Nguyen, T., Tsujiguchi, H., Kambayashi, Y., Hara, A., Miyagi, S., Yamada, Y., ... Nakamura, H. (2017). Relationship between Vitamin Intake and Depressive Symptoms in Elderly Japanese Individuals: Differences with Gender and Body Mass Index. Nutrients, 9(12), 1319. https://dx.doi.org/10.3390/nu9121319

Nogueira, S. L., Ribeiro, R. C. L., Rosado, L. E. F. P. L., Franceschini, S. C. C., Ribeiro, A. Q., \& Pereira, E. T. (2010). Fatores determinantes da capacidade funcional em idosos longevos. Brazilian 
Journal of Physical Therapy, 14(4), 322-329. https://dx.doi.org/10.1590/S1413$\underline{35552010005000019}$

Oliveira, E. M., Silva, H. S., Lopes, A., Cachioni, M., Falcão, D. V. S., Batistoni, S. S. T., Neri, A. Liberalesso, \& Yassuda, M. S.. (2015). Atividades Avançadas de Vida Diária (AAVD) e desempenho cognitivo entre idosos. Psico-USF, 20(1), 109-120. https://dx.doi.org/10.1590/1413$\underline{82712015200110}$

Oliveira, E. M., Silva, H. S., Lopes, Andrea, C., Meire, F., Deusivania, V. S., Batistoni, S. S. T., Neri, A. L., \& Yassuda, M. S. (2015). Atividades Avançadas de Vida Diária (AAVD) e desempenho cognitivo entre idosos. Psico-USF, 20(1), 109-120. https://dx.doi.org/10.1590/1413-82712015200110

Oliveira, M. F., Bezerra, V. P., Silva, A. O., Alves, M. S. C. F., Moreira, M. A. S. P., \& Caldas, C. P. (2012). Sintomatologia de depressão autorreferida por idosos que vivem em comunidade. Ciência \& Saúde Coletiva, 17(8), 2191-2198. https://dx.doi.org/10.1590/S1413-81232012000800029

Pagliai, G., Sofi, F., Vannetti, F., Caiani, S., Pasquini, G., Molino Lova, R., Cecchi, F., Sorbi, S., \& Macchi, C. (2018). Mediterranean Diet, Food Consumption and Risk of Late-Life Depression: The Mugello Study. Journal Nutrition health Aging, 22(5), 569-574. Recuperado de https://www.ncbi.nlm.nih.gov/pubmed/29717755

Paradela, E. (2011). Depressão em idosos. Revista Hospital Universitário Pedro Ernesto, 10(2) Recuperado de https://www.epublicacoes.uerj.br/index.php/revistahupe/article/view/8850/6729

Paradela, E. M. P., Lourenço, R. A., \& Veras, R. P. (2005). Validação da escala de depressão geriátrica em um ambulatório geral. Revista de Saúde Pública, 39(6), 918-923. https://dx.doi.org/10.1590/S0034-89102005000600008

Payahoo, L., Khaje-Bishak, Y., Pourghassem Gargari, B., Kabir-Alavi, M. B., \& Asgharijafarabadi, M (2013). Assessment of Nutritional and Depression Status in Free-Living El-derly in Tabriz, Northwest Iran. Health promotion perspectives, 3(2), 288-293. Recuperado de https://www.ncbi.nlm.nih.gov/pmc/articles/PMC3963672/

Pereira, G. N., Bastos, G. A. N., Del Duca, G. F., \& Bós, Â. J. G.. (2012). Indicadores demográficos e socioeconômicos associados à incapacidade funcional em idosos. Cadernos de Saúde Pública, 28(11), 2035-2042. https://dx.doi.org/10.1590/S0102-311X2012001100003

Pilger, C., Menon, M. U., \& Mathias, T. A. F. (2013). Utilização de serviços de saúde por idosos vivendo na comunidade. Revista da Escola de Enfermagem da USP, 47(1), 213-220. https://dx.doi.org/10.1590/S0080-62342013000100027

Reuben, D. B., Laliberte, L., Hiris, J., \& Mor, V. (1990). A hierarchical exercise scale to measure function at the Advanced Activities of Daily Living (AADL) Level Journal of American Geriatric Society 38(8), 855-861. https://dx.doi.org/10.1111/j.1532-5415.1990.tb05699.x

Rocha, F. S., Oliveira, P. C., \& Gardenghi, G. (2017). Perfil de idosos submetidos à avaliação geriátrica ampla em serviço de reabilitação. Revista Brasileira em Promoção da Saúde, 30(2), 170-178. Recuperado de https://periodicos.unifor.br/RBPS/article/view/5652/pdf

Rubenstein, L.Z., Harker, J.O., Salva, A., Guigoz, Y., \& Vellas, B. (2001). Screening for Undernutrition in Geriatric Practice: Developing the Short-Form Mini Nutritional Assessment (MNA-SF). The Journals of Gerontology, 56(6), 366-372. https://dx.doi.org/10.1093/gerona/56.6.M366

Santos, A. S., Ikegami, M., Almeida, J. R., Paiva, M. H. P., \& Silveira, R. E. (2016). Physical exercise and functional capacity in elderly from the south triangle health regional - Minas Gerais state, Brazil: evaluation by gender. Bioscience Journal, 32(3), 764-772. https://dx.doi.org/10.14393/J-v32n3a2016-30420

Sheikh, J.I., \& Yesavage, J.A. (1986). Escala de Depressão Geriátrica (GDS): evidências recentes e desenvolvimento de uma versão mais curta. Gerontologista Clínica: O Jornal do Envelhecimento e Saúde Mental, 5(1-2), 165-173. http://dx.doi.org/10.1300/J018v05n01 09

Silva, C. S., Barbosa, M. M. S., Pinho, L., Figueiredo, M. F. S., Amaral, C. O., Cunha, F. O., Alves, E. C. S., \& Barbosa, D. A. (2018). Estratégia saúde da família: relevância para a capacidade funcional de idosos. Revista Brasileira de Enfermagem, 71(2), 740-746. https://dx.doi.org/10.1590/0034-7167-2017-0078

Silva, V. S., Souza, I., Silva, D. A. S., Petroski, E. L., \& Fonseca, M. J. M. (2015). Correspondência entre excesso de peso e indicadores socioeconômicos e demográficos na população adulta brasileira. Revista Brasileira de Epidemiologia, 18(2), 476-489. https://dx.doi.org/10.1590/1980-5497201500020015 
Sobral, A. I. G. P., Araújo, C. M. T., \& Sobral, M. F. F. (2018). Mild cognitive impairment in the elderly Relationship between communication and functional capacity. Dementia \& Neuropsychologia, 12(2), 165-172. https://dx.doi.org/10.1590/1980-57642018dn12-020009

Tavares, D. M. S, Pelizaro, P. B., Pegorari, M. S., Paiva, M. M., \& Marchiori, G. F. (2016). Functional disability and associated factors in urban elderly: a population-based study. Revista Brasileira de Cineantropometria \& Desempenho Humano, 18(5), 499-508. https://dx.doi.org/10.5007/1980-0037.2016v18n5p499

\section{AGRADECIMENTOS}

O presente trabalho foi realizado com apoio da Coordenação de Aperfeiçoamento de Pessoal de Nível Superior - Brasil (CAPES) - Código de Financiamento 001 e pelo Instituto Ânima.

\section{CONFLITOS DE INTERESSES}

Não há conflitos de interesses.

\section{SOBRE OS AUTORES}

Amanda de Jesus dos Santos é nutricionista e Mestre em Ciências do Envelhecimento pela Universidade São Judas.

E-mail: amandajs.nutricao@hotmail.com

() https://orcid.org/0000-0002-0800-380X

Daiane Fuga da Silva é psicóloga, Mestre em Ciências do Envelhecimento pela Universidade São Judas e Docente do Curso de Psicologia da Universidade São Judas.

E-mail: daianefuga@hotmail.com

() https://orcid.org/0000-0002-9255-3694

Ana Lúcia Gatti é psicóloga, Mestre em Psicologia Clínica e Doutora em Psicologia Ciência e Profissão. E-mail: gattiana1@gmail.com

() https://orcid.org/0000-0003-2133-3843

Adriana Machado Saldiba de Lima é nutricionista e Doutora em Ciências pela Faculdade de Medicina da Universidade de São Paulo. Docente e Coordenadora do Mestrado em Ciências do Envelhecimento da Universidade São Judas.

E-mail: adrianasaldiba@gmail.com

(1) https://orcid.org/0000-0002-5741-3418

Rita de Cássia de Aquino é nutricionista, Mestre em Nutrição Humana Aplicada e Doutora em Saúde Pública pela Universidade de São Paulo. Docente na Graduação em Nutrição e no Mestrado em Ciências do Envelhecimento da Universidade São Judas.

E-mail: rcaquino@uol.com.br.

(2) https://orcid.org/0000-0002-9519-3049 